- Gardner's Syndrome (GS) should be considered whenever clinical examination reveals several palpable bony jaw swellings

- If clinical findings are suggestive of GS then a dental panoramic tomogram (DPT) is a useful initial investigation

- Early referral to a colorectal surgeon for further investigation might be life saving

\title{
Gardner's syndrome - A case report
}

\author{
M. Payne ${ }^{1}$ J. A. Anderson ${ }^{2}$ and J. Cook ${ }^{3}$
}

Gardner's syndrome is the association of multiple colonic polyps (familial adenomatous polyposis coli - FAP) with sebaceous cysts and jaw osteomas. The significance of this dominantly inherited condition to the dentist is that the colonic polyps usually undergo malignant change by the fourth decade and the extra-intestinal lesions may be apparent before those in the bowel. As such, early detection of multiple jaw osteomas and/or multiple sebaceous cysts (particularly on the scalp) may lead to appropriate further investigation and treatment which might be life saving. Diagnosis of this condition also has implications for other family members.

In February 1999 the dental panoramic tomogram (DPT) of a newly registered 28-year-old patient to a dental practice in Sheffield was sent to the radiologist at the Charles Clifford Dental Hospital for an opinion. The DPT revealed several well-defined opacities around the periphery of the mandible, and in addition demonstrated a generalised increased density to the mandible (Fig. 1). With a provisional diagnosis of Gardner's syndrome ${ }^{1}$ in mind an urgent outpatient appointment at the Dental Hospital was arranged. The patient had no complaints with regard to her jaws, but when asked directly about her bowels, admitted a four year history of diarrhoea, with occasional rectal bleeding.

On examination several bony hard swellings were palpable at the angle of the mandible bilaterally. There were no obvious cystic lesions in the soft tissues of the head and neck. In order to exclude signifi-

${ }^{1 *}$ Consultant in Dental Radiology and Assessment and Casualty, Charles Clifford Dental Hospital, Wellesley Road, Sheffield S10 2SZ; ${ }^{2}$ Consultant Surgeon, Department of General Surgery, Arrowe Park Hospital, Arrowe Park Road, Upton, Wirral, Merseyside L49 5PE; ${ }^{3}$ Consultant in Clinical Genetics, Sheffield Children's Hospital, Western Bank, Sheffield S10 2TH

Correspondence to: M. Payne

E-mail:martin.payne@sth.nhs.uk

\section{Refereed Paper}

Received 23.11.01; Accepted 03.04.02

$\odot$ British Dental Journal 2002; 193: 383-384

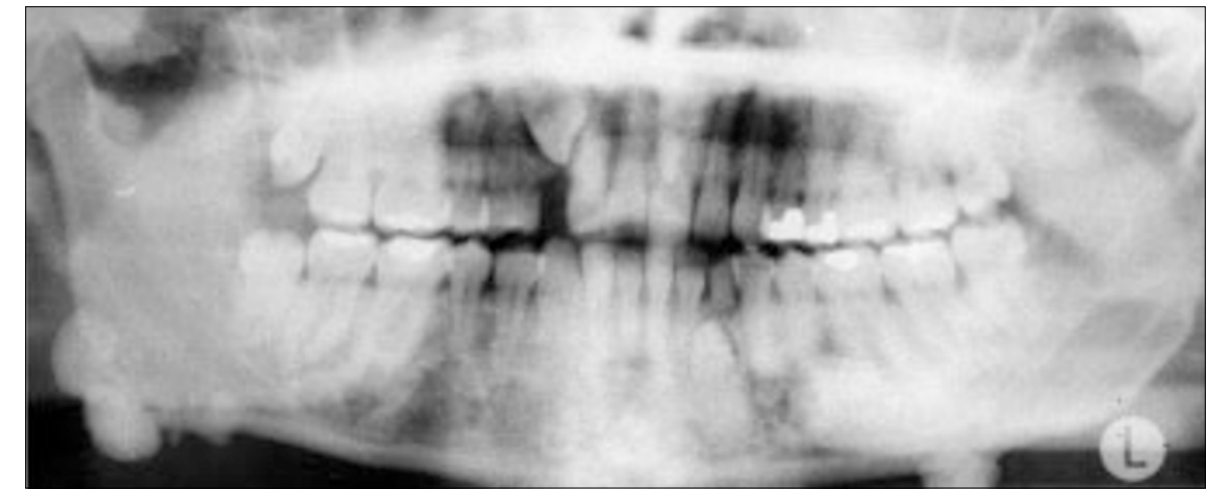

Fig. 1 DPT showing several well-defined opacities around the periphery of the mandible

cant bowel pathology she was referred for an urgent colorectal opinion.

A colonoscopy was arranged to investigate her rectal bleeding and her change in bowel habit. This revealed greater than 100 adenomatous polyps in the colon, a few of which were more than $2 \mathrm{~cm}$ in diameter, which is associated with a high risk of malignant transformation. The rectum was relatively free of polyps, and she was listed for a colectomy and ileorectal anastomosis. This surgical option avoids the formation of an ileostomy, and the morbidity of the pelvic dissection to remove the rectum, and also gives the best functional results of the bowel. The retained rectal mucosa is still susceptible to polyp formation and possible malignant change, and so she is committed to annual endoscopic surveillance of the rectum with fulguration of any developing polyps. Her operation and post-operative recovery was uneventful, and histological examination of the colon confirmed greater than 100 adenomatous polyps, none of which had undergone malignant change.

Genetics testing revealed a mutation in the adenomatous polyposis coli (APC) gene confirming the diagnosis of FAP. Identification of the specific APC mutation in this patient enables simple and accurate genetic screening of the family, so that prophylactic colectomy can be performed prior to the development of colonic cancer.

The patient's father, who was known to have a few epidermal cysts on his scalp, subsequently underwent a colonoscopy, which revealed the presence of multiple colonic polyps. 


\section{DISCUSSION}

With a prevalence of about 1 in 8,000 , familial adenomatous polyposis coli is the most common of the hereditary polyposis syndromes. ${ }^{2}$ It is caused by a dominantly inherited mutation in the APC gene localised on chromosome 5. Mutations can occur anywhere within the gene and using current technology these can be identified in about $85 \%$ of individuals with FAP by analysing a blood sample., ${ }^{3,4}$ The majority of individuals with FAP have a family history of the condition but as many as 30\% may have a new dominant mutation ${ }^{5}$ and be the first affected member of their family. The advantage of identifying the mutation in a family is that, as in this case, it allows gene carriers within the family to be accurately identified. This is particularly useful for children who, if they have not inherited the faulty gene, will never have to undergo bowel screening. In those families where a mutation cannot be identified, the only way to identify the affected individuals is by bowel screening. All family members at $50 \%$ risk have to be screened from the age of 12 . The polyps usually develop in the teenage years but if no polyps have developed by the age of 40 then screening can be discontinued.
It is important to note that polyps may develop throughout the gastro-intestinal tract. Duodenal cancer ${ }^{6}$ occurs in about 5\% of FAP patients following colectomy. Approximately 75\% of FAP patients have congenital hypertrophy of the retinal pigment epithelium (CHRPE), which is easily detected on ophthalmoscopy. It is rare for normal individuals to have more than three of these lesions, ${ }^{7}$ but there are difficulties in using the presence of CHRPE as a marker for FAP in at risk individuals because of inter-familial differences in predisposition to CHRPE. A solitary osteoma of the jaw is a common incidental finding on dental panoramic tomography, but if more than three such lesions are found this is highly suggestive of Gardner's syndrome. ${ }^{8}$

Clearly dental panoramic tomography, aside from clinical palpation, is a very effective means of detecting the multiple osteomas of the jaws that are characteristic of Gardner's syndrome. The general dental practitioner may be the first healthcare professional to suspect the diagnosis. However, it would be inappropriate to 'screen'9 every new patient to the dental practice with dental panoramic tomography for this condition, as this would represent an unnecessary financial and radiation burden to the population. However, should clinical palpation of the jaws suggest the presence of multiple osteomas then clearly a DPT would be a useful initial investigation.

1 Gardner E J, Richards R C. Multiple cutaneous and subcutaneous lesions occurring simultaneously with hereditary polyposis and osteomatosis. Am J Hum Genet 1953; 5: 139-147.

2 Alm T, Licznerski G. The intestinal polyposes. Clin Gasteroentero/ 1973; 2: 577-602.

3 Giardiello F M, Petersen G M, Piantadosi S, et al. APC gene mutations and extra-intestinal phenotype of familial adenomatous polyposis. Gut 1997; 40: 521-525.

4 Armstrong J G, Davies D R, Guy S P, Frayling I M, Evans $D$ G. APC mutations in familial adenomatous polyposis families in the north west of England. Hum Mutat 1997; 10: 376-380.

5 Bussey H J R, Veale A M O, Morson B C. Genetics of gastrointestinal polyposis. Gastroenterol 1978; 74: 1325-1330.

6 Spigelman A D, Arese P, Phillips R K S. Polyposis: the Peutz-Jeghers syndrome. BrJSurg 1995; 82: 13111314.

7 Burn J, Chapman P, Delhanty J, et al. The UK northern region genetic register for familial adenomatous polyposis coli: use of age of onset, CHRPE and DNA markers in risk calculations. J Med Genet 1991; 28: 289-296.

8 Ida M, Nakamura T, Utsunomiya J. Osteomatous changes and tooth abnormalities found in the jaws of patients with adenomatosis coli. Oral Surg 1981; 52: 2-11.

9 Rushton VE, Horner K, Worthington H V. Screening panoramic radiology of adults in general dental practice: radiological findings. Br Dent J2001; 190: 495-501. 